\title{
Effective Way to Control Acne
}

\author{
Guanting Jin ${ }^{1, *}$ \\ ${ }^{1}$ Maple Leaf International School, Dalian, Liaoning Province, 116699 \\ *Corresponding author. Email: $1444572601 @ q q . c o m$
}

\begin{abstract}
Many teenagers are suffering from acne. If the circumstance is light, only it need a few months cure. If the circumstances are serious, it will leave deep red blain prints and different shades of the pit. Acne can seriously affect the appearance and mental health of patients. There are many treatments on the market, such as laser treatment and medication. This paper would focus on these two kinds of treatment's availability. Through our research, it can be found that laser acne is the use of a specific wavelength of laser, excessive sebum and acne causing a variety of bacteria to have a certain inhibition. Retinoic acid gel can repair damaged skin so it has some effect on acne. Both methods have been proven to be effective and less harmful in clinical studies and are now commercially available.
\end{abstract}

Keywords : Acne, Retinoic acid gel, CO2 laser treatment

\section{INTRODUCTION}

In modern society, beauty is something that everyone pursues every day, with the development of The Times, cosmetics are used more and more widely. Even high school kids are starting to use touch cosmetics. However, the existence of acne will affect the psychological problems of teenagers to some extent. In this article, we will explain what acne is and how to treat it and help teenagers to solve the acne's problems to let them have more confident.

\section{INTRODUCTION TO ACNE}

Acne is a chronic inflammatory skin disease of the hair follicle sebaceous gland unit, which mainly occurs in teenagers. [4]It has a great psychological and social impact on teenagers, but it can often relieve or heal naturally after puberty. The clinical manifestations are characterized by pleomorphic skin lesions such as acne, papules, pustules and nodules.

The occurrence of acne is closely related to factors such as excessive sebum secretion, blockage of hair follicle sebaceous ducts, bacterial infection and inflammatory reaction. After puberty, the level of androgens, especially testosterone, increases rapidly, promoting the development of sebaceous glands and producing large amounts of sebum. At the same time, the hair follicle sebaceous gland catheter keratinization abnormal cause catheter blockage, sebum discharge obstacle, the formation of cutin plug is micro acne. A variety of microorganisms, especially Propionibacterium acnes, proliferate in the hair follicles. The lipase produced by propionibacterium acnes breaks down sebum to produce free fatty acids, and at the same time chemokines inflammatory cells and mediators, ultimately inducing and aggravating the inflammatory response.

Clinical feature: The non-inflammatory skin lesions of acne are characterized by open and closed acne. The typical skin lesion of closed acne is a skin papule about $1 \mathrm{~mm}$ in size with no visible hair follicle openings. Open acne is characterized by dome-shaped papules with significantly dilated hair follicle openings. Acne develops into a variety of inflammatory lesions, characterized by inflammatory papules, pustules, nodules, and cysts. Hyperpigmentation, persistent erythema, pitting or hypertrophic scars are often left after inflammatory lesions subside. Clinically, acne can be divided into four grades according to the nature and severity of acne lesions: Grade 1 (mild) : only acne; Grade 2 (moderate) : in addition to acne, there are some inflammatory papules; Grade 3 (moderate) : in addition to acne, there are more inflammatory papules or pustules; Grade 4 (severe) : in addition to acne, inflammatory papules and pustules, nodules, cysts, or scars.

Persistent acne can also leave irreversible scars. Scar is a general term for the appearance and histopathological changes of normal skin tissue caused by various wounds. It is a natural product in the process 
of wound repair. Acne is a chronic inflammatory skin disease involving the hair follicles and sebaceous glands. If the inflammation is severe or improperly handled, it can often leave shrinkage marks. Because scar is often located in the face affect the appearance. In the past, surgical methods such as grinding, surgical excision and skin grafting have been used to treat sagging graupel marks. Although they have different therapeutic effects, they have been gradually replaced by new therapeutic methods due to serious side effects such as large trauma, easy infection and even aggravation of scar. From January 2009 to April 2010, the author's department used $10600 \mathrm{~m}$ dot matrix laser to treat patients with post-acne depression scar and achieved good clinical results, as reported below.

\section{EFFECTIVE TREATMENTS}

According to the search information, found out two kinds of effective treatment of acne drugs and methods.

\subsection{Retinoic acid gel}

Studies have proved that retinoic acid itself can speed up the repair of skin lesions, when used in combination with erythromycin, its keratinolytic effect can promote the penetration of erythromycin, strengthen the control of infection, and all kinds of acne can quickly effect 2 . Amblard reported that the efficacy rate of the Chinese compound retinoic acid gel in the treatment of acne was $85 \%$, and the majority of patients achieved satisfactory efficacy in about 10 weeks, and less than $1 \%$ of patients discontinued the treatment due to poor tolerance.

It has been reported that the skin irritation symptoms of erythema, burning pain, itching and desrusting known as retinoid dermatitis may appear in the first few weeks of topical retinoic acid treatment. These symptoms will disappear after the skin adapts or can be relieved by the use of a mild moisturizer as a standard admixture. The phenomenon is considered a sign of pharmacological action rather than a toxic or allergic reaction[4].

\subsection{Laser treatment}

Facial acne is a chronic inflammatory disease of hairy feces and sebaceous glands, which occurs in adolescence. In patients, sebum production and excretion increased, hair follicle funnel keratosis, sebum excretion was obstructed, and fat plugs were formed in the hair feces, acnes bacteria in the relative hypoxia state reproduction, produce a large number of hyalidase, proteolytic enzymes, lipolytic enzymes. Destroy the hair follicle wall, glands, leading to local inflammation, suppuration and necrosis, and ultimately left depressed purpura marks. Hyperpulse carbon dioxide dot matrix laser is one of the effective methods to treat acne depression. Dot matrix laser treatment is to use the laser to hit the skin on the affected part of the average fine holes, causing a series of skin biochemical reactions, with the effect of tightening, softening and removing stains. A good effect was obtained for the patients with facial depression scar. The new holes will not overlap with each other, and the dot matrix laser treatment will only cover part of the skin tissue, maintaining normal skin and accelerating recovery. Stimulate the proliferation of collagen fibers at the base, grind and remove the sharp edge of the concave purpura mark, and make the concave part flat, so as to achieve the purpose of removing and relieving the concave purpura mark. The healing speed is accelerated and the therapeutic effect is improved[2].

The $1060 \mathrm{~m}$ lattice $\mathrm{CO} 2$ laser treatment uses the matrix pyrolysis mechanism to divide a laser beam into a number of regularly arranged microbeams, with microholes at many specific skin surface depths. Thermal stimulation of micropores can initiate tissue healing mechanism, stimulate collagen fiber proliferation and rearrangement to repair scar. Dot laser has both rapid and significant effects as an invasive treatment. It also has the advantages of less side effects and short recovery time of non-invasive treatment. It is a minimally invasive treatment between invasive and non-invasive 4 . The clinical observation results of the author showed that the total effective rate of $10600 \mathrm{~nm} \mathrm{CO} 2$ dot matrix laser in the treatment of depression scar after the fourth time was up to $8462 \%$, and with the increase of treatment times, the curative effect showed a trend of getting better and better, indicating that the effect of $\mathrm{CO} 2$ dot matrix laser in the treatment of depression scar is relatively ideal. According to pitting scar length occurred by a 1 year pitting scar can be divided into 1 year of less than 1-2 years or above 2 years into three groups for curative effect of the comparison results found no statistical significance between groups, shows that $10600 \mathrm{~m}$ of lattice co2 laser for depression effect for the treatment of scar and the scar formation of the length of time has no obvious difference with the previous literature reports the earlier treatment effect The results of the better results are different, which needs to be confirmed by larger samples of laser clinical observation and further study. The dot matrix laser creates micropores on the surface of the skin and the surrounding normal tissue acts as a bridge in the repair of the skin to ensure rapid wound healing. Therefore, the local edema and erythema immediately appeared after $\mathrm{Co} 2$ dot matrix laser are temporary and quick to recover the surface scab skin can fall off within 3 to 5 days, which has little impact on work and life, and is easier to be accepted by patients compared with the traditional treatment[3].

Results: All patients were treated 4 times after the fourth treatment. The results showed that 34 cases (52319, 21 cases were effective) had significant 
effective. Ineffective in 10 cases $(15.38 \%)$ total effective rate is $84.62 \%$. Photos of typical cases before and after

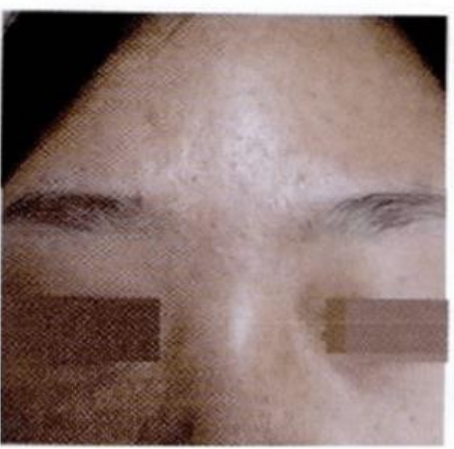

Before treatment are shown in the follow pictures.

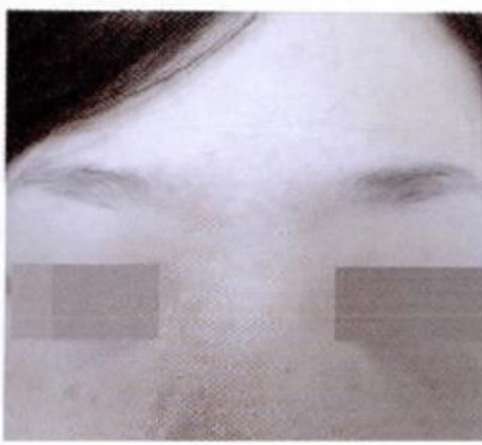

\section{After 4 times later}

Figure 1. Comparison before and after treatment

Table 1. Relationship between the length of time and treatment effect

\begin{tabular}{|c|c|c|c|c|c|c|}
\hline Time Cas & 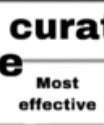 & $\begin{array}{c}\text { tive e } \\
\begin{array}{c}\text { Moderate } \\
\text { eftective }\end{array}\end{array}$ & $\frac{\text { effect }}{\text { ineffective }}$ & $\begin{array}{l}\text { Total } \\
\text { effective } \\
\text { e rate }\end{array}$ & $\begin{array}{c}\text { Test } \\
\text { statistics }\end{array}$ & $P$ \\
\hline within a year 42 & 25 & 12 & 5 & 88.10 & & \\
\hline $\mathbf{1 - 2}$ years 15 & 6 & 6 & 3 & 80.00 & $\mathrm{H}=2.618$ & 0.27 \\
\hline $\begin{array}{c}\begin{array}{c}\text { Above 2 } \\
\text { years }\end{array} \\
\end{array}$ & 3 & 3 & 2 & 75.00 & & \\
\hline
\end{tabular}

Table 2. The relationship between treatment frequency and effect

\begin{tabular}{ccccccc}
\hline Times & \multicolumn{2}{c}{ Effectiveness } & $\begin{array}{c}\text { Total } \\
\text { effective } \\
\text { effective }\end{array}$ & $\begin{array}{c}\text { Moderate } \\
\text { effective }\end{array}$ Ineffective & $\begin{array}{c}\text { Test } \\
\text { rate }\end{array}$ & statistics \\
\hline 1 & 9 & 28 & 28 & 56.92 & & \\
2 & 20 & 24 & 21 & 67.69 & $\chi^{2}=24.559$ & 0.000 \\
3 & 29 & 20 & 16 & 75.38 & & \\
4 & 34 & 21 & 10 & 84.62 & & \\
\hline
\end{tabular}

To sum up, the therapeutic effect of dot matrix laser in the treatment of sagging satin mark after acne is safe, effective, quick recovery after treatment and mild side effects bed promotion application.

\subsection{Red and blue light therapy}

At present, physical therapy such as red and blue light therapy is the best option for acne patients who cannot tolerate medication or do not want to take medication for a long time. In recent years, red and blue light therapy, as a new physiotherapy, has pointed out a new direction for the treatment of acne. Studies have shown that the application of red and blue light combined with $0.1 \%$ Adapalene gel can achieve satisfactory therapeutic effect. Red and blue light are produced by light emitting diodes (leds), whose main advantage is that they produce a high purity narrow spectrum of blue and red light, filtered by ultraviolet and other doped elements. Acne is mainly caused by propionibacterium acnes infection, and the most suitable wavelength for damaging acne propionibacterium infection is in the blue region. This study on the application of wavelength is $460 \mathrm{~m}$ blue-ray and wavelength is $630 \mathrm{~m}$ red combined treatment of acne, $460 \mathrm{~nm}$ blue light activated acne propionic acid bacillus metabolism of endogenous photosensitizer porphyrin, produce light poison environment, transformation of toxic singleton reactive oxygen species quickly kill acne propionic acid bacillus, and does not harm the other normal skin tissue, killing 
acne propionic acid bacillus, which in turn Clear acne. Meanwhile, blue light can also inhibit the proliferation of propionibacterium acnes by inducing changes in cell membrane permeability and $\mathrm{pH}$ value of cells, thus inhibiting the inflammatory response of acne. Studies by Fukao et al. have shown that blue light can also reduce the number of propionibacterium acneae. Narrow blue light can inhibit the inflammatory response of cells. Since the penetration depth of blue light is $1 \sim 2 \mathrm{~m}$, blue light only acts on the skin epidermis, but cannot effectively penetrate deeper tissues. And when red light irradiation in the $630 \mathrm{~m}$, penetration depth can reach 6 $\mathrm{mm}$ can penetrate deeper organizations such as leather, can pass the light effect, the activation energy of the switch on the mitochondria, the activation cell function to absorb energy not only can stimulate macrophages and other cells of anti-inflammatory cytokine, achieve the effect of anti-inflammatory, and can promote the synthesis of collagen dermal papilla layer increases, the change It can improve the microcirculation, stimulate the activity of mitochondria in cells, accelerate the repair of damaged tissues, and significantly reduce the formation of acne moving marks. Therefore, the use of alternating red and blue light irradiation than a single blue or red light, in the treatment of acne, more advantages. Therefore, it can be used to clear picea so as to reduce the inflammatory lesions of acne such as papules, purpus and cysts as well as scar formation in acne. It can also promote the repair of pure sore scar, fade scar, beautiful and tender skin, achieve the effect of beauty, and painless, non-invasive, less side effects. As a synthetic third generation retinoic acid, $0.1 \%$ adapalene is a stable naphthalic acid derivative. It not only inherits the pharmacological activity of traditional retinoic acid drugs, but also has stronger anti-inflammatory activity and better stability. The topical application of $0.1 \%$ adapalene is less irritating than $0.025 \%$ tretinoin. It can not only inhibit sebum secretion, but also improve the keratosis embolization of sebaceous ducts in the hair follicles of acne patients, dissolution of acne, prevent the formation of new micro-acne, and completely remove the environment favorable for propionibacterium acne propagation. The $0.1 \%$ adapalene gel has good permeability and a high concentration of the drug in the keratin. It has a good therapeutic effect on both inflammatory and non-inflammatory skin lesions of acne. The application of red and blue light combined with $0.1 \%$ adapalene gel in the treatment of acne has a significant effect, which makes up for the deficiency of single drug therapy, and has fewer adverse reactions. Patients' compliance and satisfaction rate were improved. It is a safe and effective method for the treatment of acne and is worthy of clinical recommendation[1].

\section{CONCLUSION}

In conclusion, this paper studied the effectiveness of laser therapy, drug therapy and red-blue light therapy on acne. The conclusion is that both of them can solve the acne problem to some extent and inhibit the production of acnebacter. Patients can choose the most suitable regimen according to the severity of their acne and the need for recovery time.

\section{ACKNOWLEDGMENTS}

First, I am very grateful to my two advisors for helping me check the article and giving me suggestions for revision. The teachers also discuss about the structure of the article with me, which inspires me a lot. Second, I am very grateful to the relevant medical personnel for their investigation and reference. Without their help, I cannot finish the report.

\section{REFERENCES}

[1] Ji, Li. Clinical observation of 134 cases of acne treated with red and blue light combined with $0.1 \%$ adapaline gel. ixueshu. Chinese Medical cosmetology. n.p. 57

[2] Liqiu, Song. Effect of $\mathrm{Co} 2$ dot array laser on depression scar after acne. ixueshu. Chinese Medical cosmetology. Aug, 2013. 1620

[3] Fusheng, Han. Effect of ultra-pulsed Co2 dot array laser on depression scar after acne. Chinese Medical cosmetology. 2016. 53

[4] Aiping, Wang. Efficacy and safety of compound retinoic acid gel in the treatment of acne vulgaris. ixueshu. Journal of Practical Dermatology

[5] Hui, Lin. Clinical effect and safety analysis of fruit acid skin changing combined with adapaline gel in the treatment of mild to moderate acne. wanfangdata. Chinese and Foreign medical research. July, 2020

[6] Yanfeng Xu. Effect of red and blue light combined with Adapalin gel on acne. wanfangdata. China Medical Cosmetology. July, 2020 